\title{
Propuesta, indicadores de movilidad sostenible para la ciudad de Cuenca, Ecuador
}

Proposal, sustainable mobility indicators for the city of Cuenca, Ecuador

1 Christian Fabián Guerrero Dumas https://orcid.org/0000-0001-7145-2985

Universidad Católica de Cuenca, Maestría en Construcciones con mención en

Administración de la Construcción Sustentable, Azuay,

christian.guerrero.75@est.ucacue.edu.ec

2 César Maldonado Noboa

https://orcid.org/0000-0003-0383-5460

Universidad Católica de Cuenca, Maestría en Construcciones con mención en

Administración de la Construcción Sustentable, Azuay

cmaldonadon@ucacue.edu.ec

3 Deisy Katerine Reyes Rodas

https://orcid.org/0000-0003-1348-1897

Universidad Católica de Cuenca, Carrera de Arquitectura, Azuay,

dreyesr@ucacue.edu.ec

4 Marco Ávila Calle

https://orcid.org/0000-0002-2134-1432

Universidad Católica de Cuenca, Carrera de Arquitectura, Azuay,

mavila@ucacue.edu.ec

Artículo de Investigación Científica y Tecnológica

Enviado: 07/12/2021

Revisado: 22/12/2021

Aceptado: $17 / 01 / 2022$

Publicado:05/03/2022

DOI: https://doi.org/10.33262/concienciadigital.v5i1.2.2085

Cítese: $\quad$ Guerrero Dumas, C. F., Maldonado Noboa, C., Reyes Rodas, D. K., \& Ávila Calle, M. (2022). Propuesta, indicadores de movilidad sostenible para la ciudad de Cuenca, Ecuador. ConcienciaDigital, 5(1.2), 46-65.

https://doi.org/10.33262/concienciadigital.v5i1.2.2085

CONCIENCIA DIGITAL, es una Revista Multidisciplinar, Trimestral, que se publicará en soporte electrónico tiene como misión contribuir a la formación de profesionales competentes con visión humanística y crítica que sean capaces de exponer sus resultados investigativos y científicos en la misma medida que se promueva mediante su intervención cambios positivos en la sociedad. https://concienciadigital.org La revista es editada por la Editorial Ciencia Digital (Editorial de prestigio registrada en la Cámara Ecuatoriana de Libro con No de Afiliación 663) www.celibro.org.ec 


\section{Palabras} claves:

Sostenibilidad; transporte; movilidad sostenible; indicadores de movilidad sostenible.

\section{Keywords:}

Sustainability; transportation; sustainable mobility; sustainable

\section{Resumen}

Introducción. El desarrollo sostenible hoy en día juega un papel importante en las ciudades que poseen la particularidad de ser economías de rápido crecimiento y poblaciones de tamaño intermedio como es la ciudad de Cuenca. Entre varios objetivos que tiene el desarrollo sostenible, la siguiente investigación de carácter exploratoria-descriptiva se enfoca en la movilidad sostenible. Objetivo. Con el objetivo de tener una propuesta de indicadores, se efectuó una revisión y análisis de las siguientes guías metodológicas: 1. 3. ${ }^{a}$ Edición BID: Iniciativa Ciudades Emergentes y Sostenibles., 2. ISO 37120: Desarrollo Sostenible de Comunidades y 3. El Objetivo 11 del Desarrollo Sostenible, documentos de base que son el punto de partida para la presente investigación. De igual manera se estudió las siguientes herramientas de evaluación: 1. BREEAM (BRE Environmental Assessment Method), 2. CASBEE (Comprehensive Assessment System for Building Environmental Efficiency) y 3. LEED v4.1 (Leadership in Energy \& Environmental Design). Como complemento se realizó una revisión de un caso de estudio denominado: Evaluación del transporte urbano e impactos de COVID-19 en movilidad de las Naciones Unidas ESCAP. Metodología. La determinación de la propuesta de indicadores se efectúo a través de una puntuación con criterios de: suficiencia, claridad, relevancia y coherencia, realizada por un juicio de expertos, mismos que analizaron y calificaron estos. Resultados. Llegando a proponer un total de 16 indicadores, estos se obtuvieron de la homologados de 43 que fueron seleccionados del estudio inicial de la bibliografía mencionada. Conclusiones. Todos los indicadores propuestos son de importancia, ya que la gran mayoría alcanzo una alta puntuación según los criterios mencionados. Se evaluó 3 indicadores de relevancia los mismos que en base a su valor calculado permitieron la apreciación del cumplimiento de sostenibilidad, de una sostenibilidad media y no sostenibilidad en la ciudad de Cuenca.

\section{Abstract}

Introduction. Today, sustainable development plays an important part in cities that have the particularity of being fast-growing economies and medium-sized populations, such as the city of Cuenca. Among several goals of sustainable development, the 
mobility indicators. following exploratory-descriptive research focuses on sustainable mobility. Goal. To have a proposal for indicators, a review and analysis of the following methodological guidelines was conducted: 1. 3. ${ }^{\mathrm{a}}$ Edition BID: Emerging and Sustainable Cities Initiative., 2.ISO 37120: Sustainable Development of Communities and 3. Sustainable Development Goal 11, basic documents that are the starting point for this research. In the same way, the following evaluation tools were studied: 1. BREEAM (BRE Environmental Assessment Method), 2. CASBEE (Comprehensive Assessment System for Building Environmental Efficiency) y 3. LEED v4.1 (Leadership in Energy \& Environmental Design). As a complement, a review of a case study called: Evaluation of urban transport and impacts of COVID-19 on mobility of the United Nations ESCAP was also conducted. Methodology. The determination of the proposal of indicators was conducted through a score with criteria of sufficiency, clarity, relevance, and coherence, this was made out by a judgment of experts, who did the analysis and put the score. Results. Proposing a total of sixteen indicators, these were obtained from the approved forty-three that were selected from the initial study of the mentioned bibliography. Conclusions. All the proposed indicators are important since the majority achieve a high score according to the highlighted criteria. Three indicators of relevance were evaluated, the same ones that, based on their calculated value, allowed the appreciation of sustainability compliance, medium sustainability, and nonsustainability in the city of Cuenca.

\section{Introducción}

De acuerdo a la Organización de Naciones Unidas (ONU), se define al desarrollo sostenible como: "Satisfacer las necesidades de la generación presente sin comprometer la capacidad de las generaciones futuras para satisfacer sus propias necesidades" (Brundtland, 1987, p.23). Luego según Manzanares Garmendia (2020):

En 1992, la comunidad internacional se reunió en Río de Janeiro, Brasil, para discutir los medios para poner en práctica el desarrollo sostenible. Los líderes mundiales adoptaron el Programa 21, con planes de acción específicos para lograr el desarrollo sostenible en los planos nacional, regional e internacional. Esto fue seguido en 2002 por la Cumbre Mundial sobre el Desarrollo Sostenible, donde se 
aprobó el Plan de Aplicación de Johannesburgo, dicho plan se basó en los progresos realizados y las lecciones aprendidas desde la Cumbre de la Tierra, y prevé un enfoque más específico, con medidas concretas, plazos y metas cuantificables. (p. 5)

La Declaración de Johannesburgo afirma que: "la integración de los tres componentes del desarrollo sostenible —el crecimiento económico, el desarrollo social y la protección del medio ambiente-, son pilares interdependientes que se refuerzan mutuamente" (Engström et al., 2002, p.8). Asimismo, según Manzanares Garmendia (2020), "en el documento más reciente de la ONU en el año 2012, acerca del desarrollo sostenible se reafirma el énfasis en la integración multidimensional” (p.5).

En ese orden de ideas, en el 2015 los Objetivos de Desarrollo Sostenible (ODS) se convierten en el marco de referencia para dar operatividad al desarrollo sostenible y contribuir a la integración multidimensional que desde años atrás se venía proponiendo. En ese sentido,

- El Programa de las Naciones Unidas para el Desarrollo PNUD (como se citó en Manzanares Garmendia, 2020) son un llamado universal para poner fin a la pobreza, proteger el planeta y garantizar que todas las personas gocen de paz y prosperidad para el 2030.

El término de desarrollo sostenible ha sido políticamente identificado como un papel importante para planificación espacial. "La necesidad de desarrollar estrategias que ayuden a las comunidades a avanzar hacia la sostenibilidad de manera económica, social y ambiental prevalece en el debate e investigación de la planificación contemporánea y práctica” (Mascarenhas, Nunes, Ramos y Tomás, 2015, p. 1).

En base a lo mencionado por Gudmundsson et al. (2016), "la sostenibilidad se ha convertido en una preocupación urgente para la política de transporte y planificación de movilidad en todo el mundo. El concepto de transporte sostenible está ampliamente definido, lo que permite que se establezcan políticas y prácticas" (p.1). "Las Naciones Unidas para el 2050 el $66 \%$ de la población mundial vivirá en zonas urbanas lo que dará lugar a grandes desafíos relacionados con la contaminación del aire, la congestión, gestión de residuos y salud humana" (Ahvenniemi et al., 2017, p. 234). Considerando que los desafíos de la movilidad de las ciudades en desarrollo, en este caso los procedentes de Asía, China, India, África y Sudamérica son diferentes a las de los países más ricos y avanzados, tenemos que según Cervero (2013):

Las características cardinales del transporte, el urbanismo integrado y sostenible en todas partes, actividades urbanas accesibles, entornos seguros, atractivos para 
caminar y andar en bicicleta son particularmente vitales para el bienestar y la prosperidad de los habitantes en zonas urbanas de los países en desarrollo. (p. 7)

En base al Plan de Movilidad y Espacios Públicos, según la Ilustre Municipalidad de Cuenca (GAD Municipal, 2015) menciona que:

La Plataforma de las Ciudades Emergentes y Sostenibles, desarrollada por el BID analizan a 140 ciudades de Sudamérica y El Caribe, que poseen la particularidad de ser economías de rápido crecimiento y poblaciones de tamaño intermedio, dentro de este estudio se encuentra considerada la ciudad Cuenca. (p. 210)

En base a los mencionado anteriormente, la Organización Internacional de Normalización (ISO, 2014) "para rastrear y monitorear el progreso en el desempeño del servicio de la ciudad y la calidad de vida definen indicadores que permiten ayudar a las ciudades a establecer objetivos y monitorear los logros y metas alcanzadas" (p. 3). A pesar de la importancia que representan los indicadores en la planificación de la movilidad, en base a Flores, García, Chica y Mora (2017), “en Ecuador no se encuentran desarrollados con claridad dichos temas, situación que demuestra la importancia de identificar y analizar indicadores que permitan medir dicho fenómeno" (p. 100 ), los mismos que una vez propuestos en base al estudio y análisis de guías metodológicas y herramientas de evaluación, servirán a la institución pública encargada como instrumento de aporte en la toma de decisiones al momento de planificar proyectos de movilidad.

La presente investigación tiene el objetivo de revisar y analizar las guías metodológicas como: 1. 3. ${ }^{a}$ Edición BID: Iniciativa Ciudades Emergentes y Sostenibles, 2. ISO 37120 : Desarrollo Sostenible de Comunidades y 3. El Objetivo 11 del Desarrollo Sostenible. También herramientas de evaluación como: 1. BREEAM (BRE Environmental Assessment Method); 2. CASBEE (Comprehensive Assessment System for Building Environmental Efficiency) y 3. LEED v4.1 (Leadership in Energy \& Environmental Design). Así también se ha revisado y analizado un caso de estudio titulado: Evaluación del transporte urbano e impactos de COVID-19 en movilidad de las Naciones Unidas ESCAP, con la finalidad de generar una propuesta de indicadores de movilidad sostenible que puedan aplicarse para la ciudad de Cuenca.

\section{Movilidad sostenible.}

El desarrollo del concepto de movilidad sostenible según Damidavičius et al. (2020):

Hace hincapié en la planificación integrada de los sistemas de transporte, y presta especial atención a la expansión del transporte público y no motorizado, de igual manera a los diferentes sistemas compartidos, así como a la gestión eficaz del tráfico que implica sistemas de transporte inteligentes. (p.1) 
- El Consejo Empresarial Mundial para el Desarrollo Sostenible (como se citó en Gillis et al., 2015), formula el proceso de desarrollo de la movilidad urbana sostenible de la siguiente manera: El objetivo final es acelerar y ampliar el acceso a una movilidad segura, confiable y cómoda para todos sin tener que sufrir accidentes de tráfico, tener un bajo impacto ambiental, ser asequible y reducir las demandas de energía y tiempo.

Analizando planes de movilidad sostenible en España hoy en día es un desafío, teniendo como objetivos según Mozos-Blanco et al., (2018) "no solo la reducción de la congestión y la contaminación urbana, sino animar a los ciudadanos a que logren ser menos dependientes del coche y más activos en sus desplazamientos diarios” (p.45).

Los viajes de trabajo son los que más contribuyen a la congestión durante las horas pico en las áreas urbanas. Por esta razón, menciona Guzmán et al. (2020) "que las organizaciones públicas y privadas pueden desempeñar un papel relevante desarrollando planes de movilidad para proponer posibles estrategias que afronten la congestión" (p.321).

\section{Indicadores de sostenibilidad.}

En efecto, estamos frente a un cambio de época: "la opción de continuar con los mismos patrones de producción, energía y consumo no es viable, es necesario transformar el paradigma de desarrollo dominante por uno del desarrollo sostenible, inclusivo y con visión de largo plazo" (Parra Cortés, 2018, p.7).

Los indicadores se utilizan ampliamente para evaluar el progreso, los proyectos y políticas para establecer metas y objetivos. La Organización para la Cooperación y el Desarrollo Económico define los indicadores como medidas estadísticas de sostenibilidad social, ambiental y económica. “ Un conjunto de indicadores apropiados permite tomar decisiones responsables para monitorear el estado y comprender las consecuencias de las acciones e inacciones" (Jain \& Tiwari, 2017, p.310).

\section{Metodología}

En base a la bibliografía estudiada se sabe que el transporte juega un rol muy importante en las ciudades emergentes para alcanzar la sostenibilidad de manera social, económica y ambiental. Por tal motivo se seleccionaron varios indicadores, los mismos que servirán como propuesta para monitorear y conocer el estado de la movilidad en la ciudad, logrando de esta manera definir criterios a ser evaluados previa elaboración de proyectos referentes a movilidad sostenible. La siguiente investigación se centra en un enfoque investigativo descrito en la tabla 1. 


\section{Tabla 1}

Tipo y Nivel de Investigación

Según su finalidad

Básica

Según su alcance temporal

Longitudinal prospectiva

Mejoró el conocimineto y comprensión de indicadores para una movilidad sostenible en la ciudad de Cuenca.

La propuesta de indicadores se prentende evaluar con datos cercanos al presente, con el objetivo de la proyección de resultados a fututo.

En la siguiente investigación se exploró guías metodológicas, herramientas de evalaución y un

Según su profundidad
Exploratoría -

Descriptiva caso de estudio sobre indicadores para una movilidad sostenible.

\begin{tabular}{ll}
\hline $\begin{array}{l}\text { Según las } \\
\text { fuentes }\end{array}$ & Mixtas \\
\hline $\begin{array}{l}\text { Según el } \\
\text { carácter }\end{array}$ & $\begin{array}{l}\text { Cualitativas y } \\
\text { Cuantitativas. }\end{array}$ \\
\hline $\begin{array}{l}\text { Según su } \\
\text { naturaleza }\end{array}$ & $\begin{array}{l}\text { Documentales } \\
\text { y Encuestas }\end{array}$ \\
\hline
\end{tabular}

Debido a que la información obtenida fue de fuentes primarias y secundarias.

Los resultados obtenidos se basa en datos cualitativos y la evaluación en cuantitativos.

Mediante fuentes secundarías se propone los indicadores, mismos que en base a fuentes primarias como el juicio de expertos fuerón seleccionado de acuerdo a su relevancia.

Debido al tipo y nivel de investigación, la misma se divide en tres fases como se observa en la figura 1.

\section{Figura 1}

Proceso de selección para la propuesta de indicadores para una movilidad sostenible en la ciudad de Cuenca
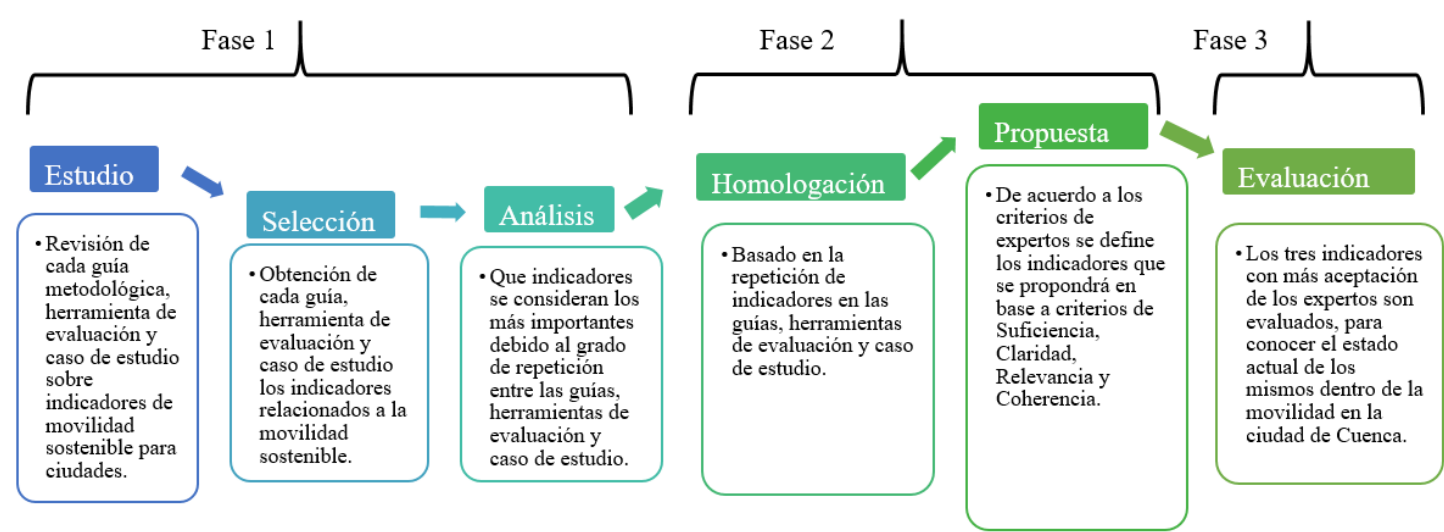

Nota: Proceso de investigación para la propuesta y evaluación de indicadores de movilidad sostenible. 
Fase 1: A través de fuentes secundarías como las: guías metodológicas, herramientas de evaluación y caso de estudio se revisó, estudió y analizó lo relacionado a movilidad sostenible, documentos que permiten la generación de una primera selección de indicadores relevantes comunes en la mayoría de estos y de aplicación para ciudades con condiciones parecidas a la ciudad de Cuenca, principalmente; basados en aspectos económicos, sociales y ambientales, los mismos que son propuestos y evaluados en la fase 2 que hace referencia al juicio de expertos.

Fase 2: Según Pérez \& Martínez (2008), "definen al juicio de expertos como una opinión informada de personas con una gran trayectoria en el tema” (p. 29). Ante ello los criterios de inclusión utilizados para conformar el grupo de expertos se basó en su perfil y las actividades profesionales relevantes que cada uno desempeña.

Para la selección del grupo de expertos se elaboró una encuesta digital a 15 profesionales vinculados con proceso y temas de movilidad y que trabajan en entidades o para ellas directamente con proyectos relacionados, el objetivo principal fue asegurar el grado de conocimiento que los profesionales tienen en estudios o proyectos referentes a movilidad, tránsito y transporte, lo que permite la propuesta de los indicadores para una movilidad sostenible en la ciudad de Cuenca. Se llegó a seleccionar cinco expertos, quienes, en base a su experiencia profesional y perfil académico, basados en criterios de: suficiencia, claridad, relevancia y coherencia, establecieron una calificación cualitativa a cada indicador.

Fase 3: En esta fase se evaluó 3 indicadores propuestos que obtuvieron una alta puntuación en la fase 2, y de esta manera determinar si la ciudad está desarrollándose y creciendo, considerando y priorizando estrategias a escala local y nacional que logren sociedades modernas futuras, promoviendo los desplazamientos de peatones, personas y carga en condiciones y de forma eficiente, segura y amigable.

\section{Resultados}

Del estudio bibliográfico de las guías metodológicas, herramientas de evaluación y caso de estudio para la movilidad sostenible se obtuvo un total de 43 indicadores los mismos que pueden ser observados en la Figura 2, y descritos cada uno de estos en la figura 2. 


\section{Figura 2}

Cantidad de indicadores por cada documento

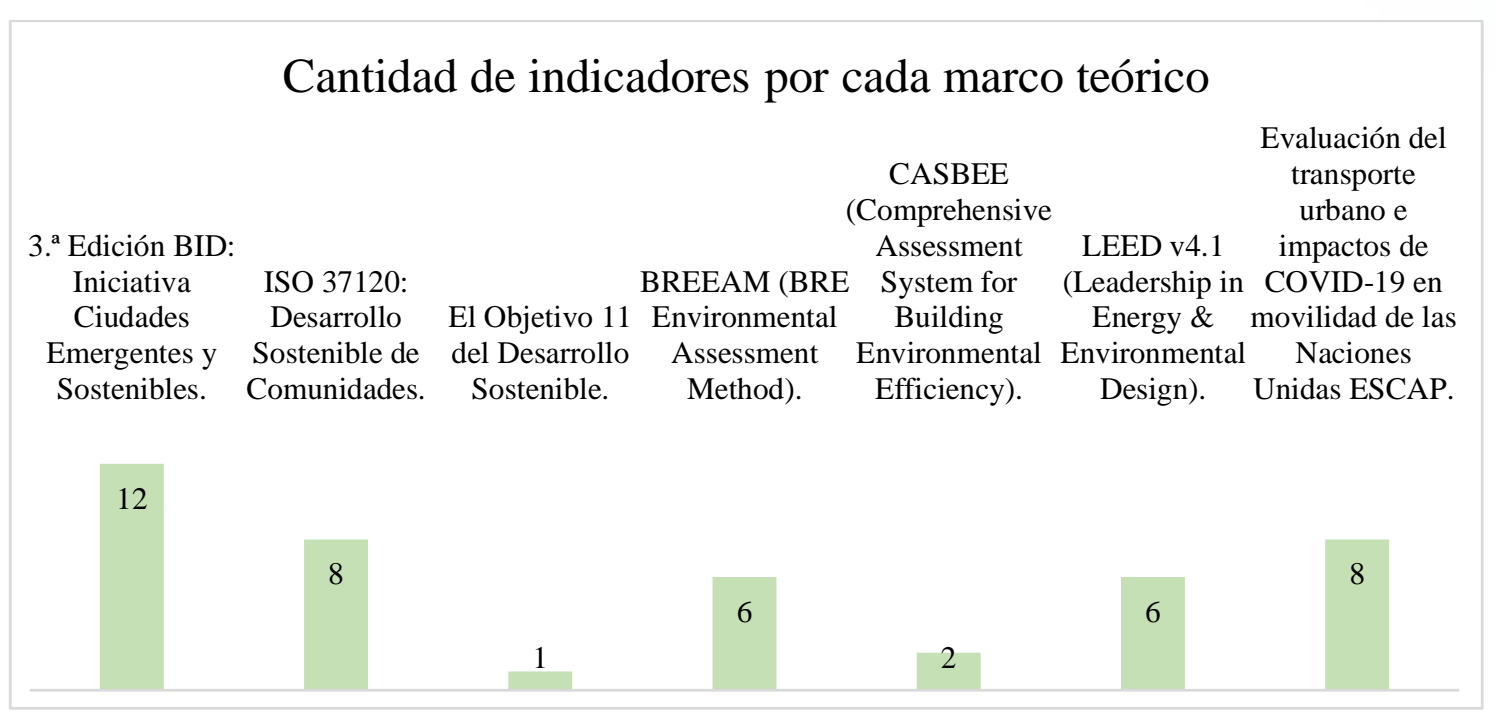

Nota: Indicadores por cada guía metodológica, herramienta de evaluación y casos de estudio.

Fuente: Guía Metodológica BID (2016), Desarrollo sostenible de comunidades (ISO 37120, 2014), El objetico 11 del desarrollo sostenible (UN-Hábitat, 2019), Communities technical manual (BREEAM, 2012), Technical manual (CASBEE, 2012), Ciudades y comunidades: Planificar y diseñar (LEED V4, 2020) y Evaluación del transporte urbano e impactos de COVID-19 en movilidad (United Nations ESCAP, 2020). 


\section{DDigital}

ISSN: 2600-5859

Vol. 5 No. 1.2 , pp. $46-65$, marzo 2022

www.concienciadigital.org

\section{Figura 3}

Indicadores referentes a la movilidad sostenibles (Guías metodológicas, herramientas de evaluación y caso de estudio)

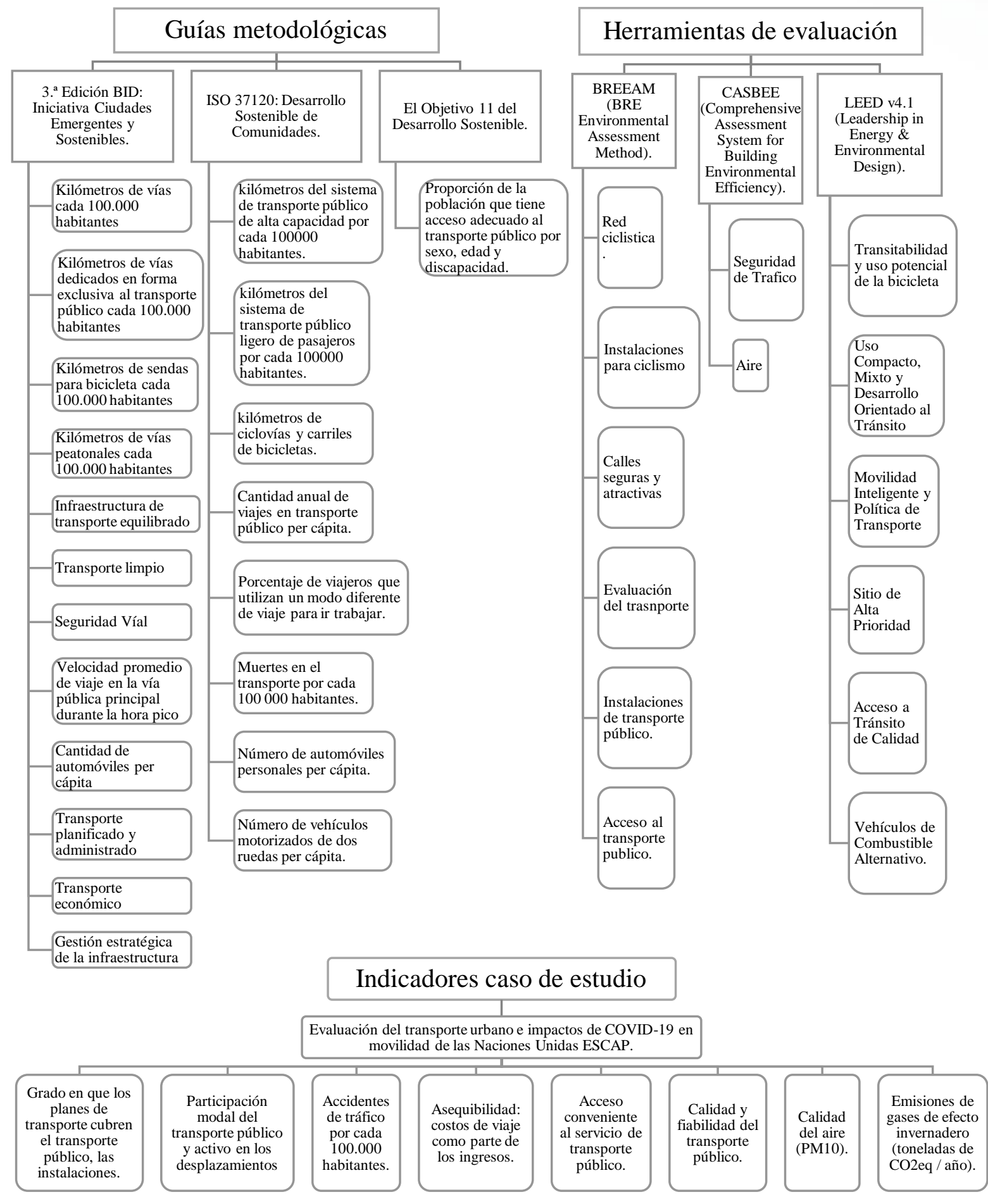


De acuerdo con el proceso de análisis de las fuentes secundarias documentales, se conoce que la sostenibilidad debe estar enfocada en aspectos económicos, sociales y ambientales. En base al estudio de las guías metodológicas, herramientas de evaluación y caso de estudio se pudo notar que en las bibliografías analizadas dan mayor importancia al aspecto social, seguido del aspecto ambiental y finalmente el aspecto económico como se puede observar en la figura 4.

\section{Figura 4}

Cantidad de indicadores de movilidad sostenible basados en aspectos sociales, ambientales y económicos

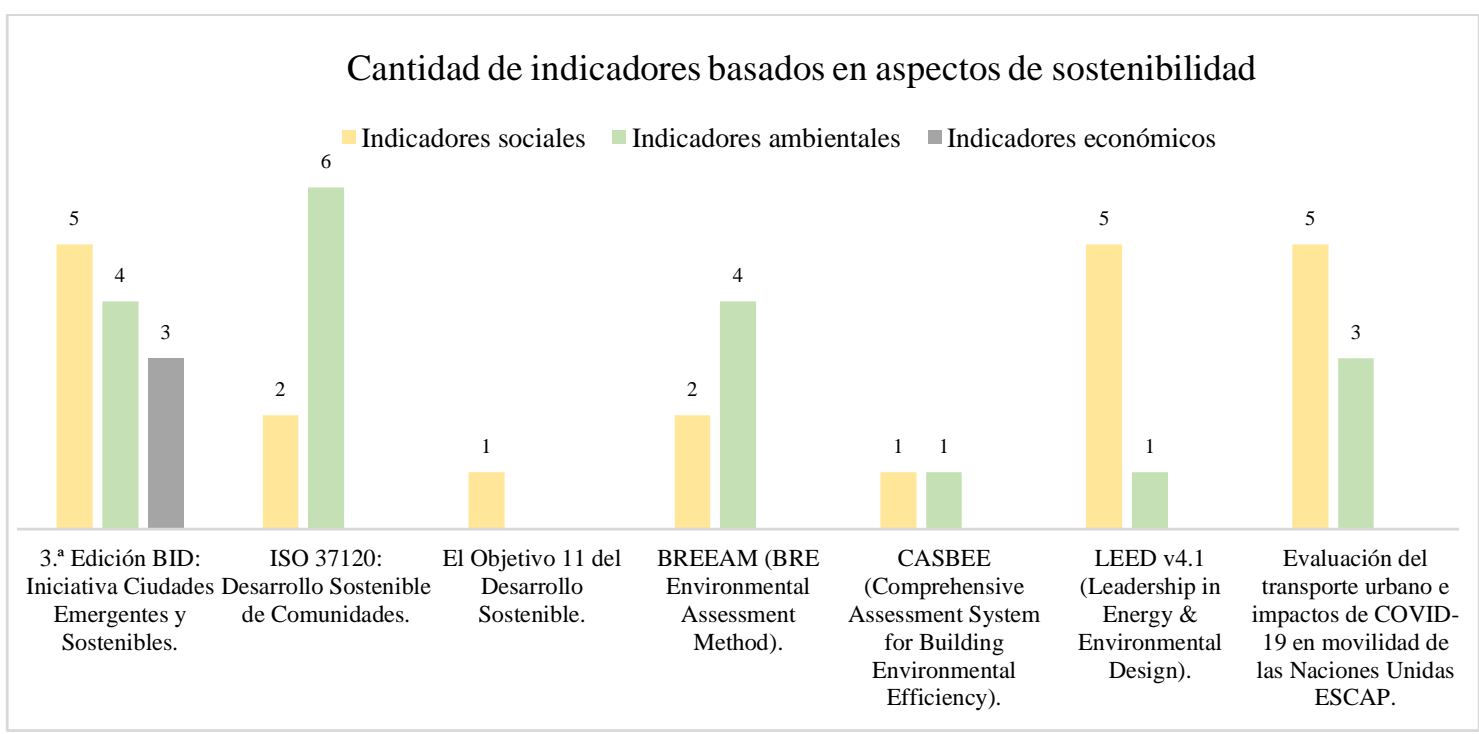

Nota: Enfoque de cada guía metodológica, herramienta de evaluación y caso de estudios en aspectos sociales, ambientales y económicos.

Para una primera selección (Fase 1) de los indicadores sobre la movilidad sostenible, se realizó una homologación entre los 43 indicadores obtenidos en las guías metodológicas, herramientas de evaluación y caso de estudio como se observa en la figura 3, llegando a proponer para la presente investigación 16 indicadores que contienen la mayoría los mencionados en las fuentes secundarias de revisión, cabe indicar que en muchos casos cuentan con nombres diferentes, pero con el mismo objetivo por lo que su homologación se hizo en un solo indicador. 


\section{Conciencia}

\section{Figura 5}

Homologación de indicadores obtenidos de las guías metodológicas, herramientas de evaluación y caso de estudio

1. Infraestructura de vías.

2. Vías de sistema de transporte público.

\section{Ciclovías.}

\section{Vías peatonales.}

5. Porcentaje de viajes en transporte público per cápita.

\section{Edad de flota vehícular.}

\section{Accidentes de tránsito.}

8. Velocidad promedio del transporte.

9. Cantidad de automóviles personales per cápita.

10. Políticas públicas del transporte.

11. Valor mensual de transporte por persona.

12. Gestión estratégica de la infraestructura.

13. Cantidad de vehículos motorizados de dos ruedas per cápita.

14. Acceso al servicio de transporte público.

15. Emisiones de gases de efecto invernadero.

16. Cantidad de vehículos de combustible alternativo.
- Infraestructura de transporte equilibrado-kilómetros de vías cada 100.000 habitantes.

- Infraestructura de transporte equilibrado alta capacidad-kilómetros de vías cada 100.000 habitantes

dedicadas al transporte público.

- Infraestructura de transporte equilibrado ligera capacidad-kilómetros de vías cada 100.000 habitantes

dedicadas al transporte público.

- kilómetros del sistema de transporte público.

- Los kilómetros de línea dedicados a la bicicleta.

- kilómetros de ciclovías y carriles de bicicletas.

- Transitabilidad y Uso potencial de la Bicicleta.

- Red ciclistica.

- Diseñando los detalles Instalaciones para ciclismo.

- Grado en que los planes de transporte cubren el transporte público, las instalaciones.

- Infraestructura de transporte equilibrado-kilómetros de vías peatonales cada 100.000 habitantes - Calles seguras y atractivas.

Infraestructura de transporte equilibrado.

- Cantidad anual de viajes en transporte público per cápit

- Porcentaje de viajeros que utilizan un modo diferente de viaje para ir trabajar.

- Uso Compacto, Mixto y Desarrollo Orientado al Tránsito.

- Participación modal del transporte público y activo en los desplazamientos.

- Transporte limpio.

- Seguridad Víal.

Muertes en el transporte por cada 100.000 habitantes.

- Seguridad de Trafico.

- Accidentes de tráfico por cada 100.000 habitantes.

- Congestión reducida-velocidad promedio de viaje.

- Congestión reducida-cantidad de automóviles per cápita.

- Número de automóviles personales per cápita.

- Transporte planificado y administrado.

- Movilidad Inteligente y Política de Transporte.

- Sitio de Alta Prioridad.

- Determinando el diseño del desarrollo.

- Transporte económico

- Asequibilidad: costos de viaje como parte de los ingresos

- Gestión estratégica de la infraestructura.

- Instalaciones de transporte público

- Número de vehículos motorizados de dos ruedas per cápita.

- Proporción de la población que tiene acceso adecuado al transporte público por sexo, edad discapacidad.

- Acceso a Tránsito de Calidad.

- Acceso al transporte publico.

- Acceso conveniente al servicio de transporte público.

- Acceso conveniente al servicio de transpor y fiabilidad del transporte público.

- Aire.

Calidad del aire (PM10).

Emisiones de gases de efecto invernadero (toneladas de $\mathrm{CO} 2 \mathrm{eq} /$ año)

- Vehículos de Combustible Alternativo.

Para la fase 2 se elaboró una: "guía para la evaluación del juicio de expertos" (Pérez \& Martínez, 2008, pp.30-31), teniendo como puntos principales: a. La definición del 
objetivo para el juicio de expertos, b. Elección de expertos, c. Informar sobre los indicadores d. Definir objetivo de la encuesta. e. Establecer puntuaciones. f. Elaboración de encuestas. g. Mayor puntuación por los jueces. h. Generar conclusiones en base a los resultados obtenidos. Con el objetivo de definir a los expertos enrolados en área de la movilidad, se elaboró una encuesta digital a varios profesionales, obteniendo los resultados que se observan en la figura 6 .

\section{Figura 6}

Resultados de la encuesta digital para la selección de expertos en temas de movilidad dentro de la ciudad de Cuenca

\begin{tabular}{|c|c|c|c|c|c|c|c|c|c|c|c|c|c|c|c|c|c|c|c|c|c|c|}
\hline & Proyectos y estud & ios re & lacion & ados & a los & indica & adores & es pro & puest & tos par & ira un & a moy & vilidas & d sost & tenibl & & & & & & & \\
\hline No. & \begin{tabular}{|l|} 
Profesionales \\
encuestados con \\
conocimientos en el \\
área de movilidad \\
para la ciudad de \\
Cuenca.
\end{tabular} & 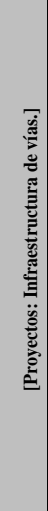 & 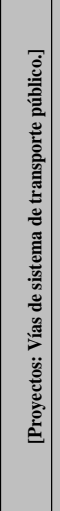 & 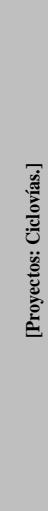 & 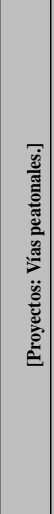 & 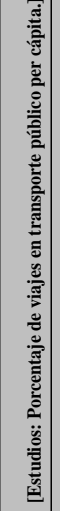 & 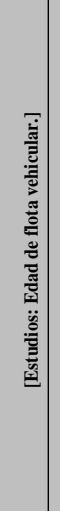 & 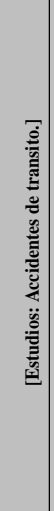 & 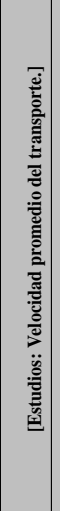 & 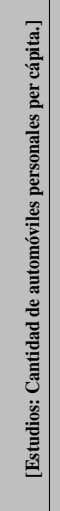 & 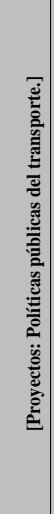 & 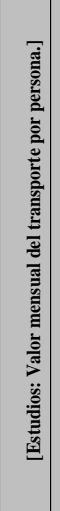 & 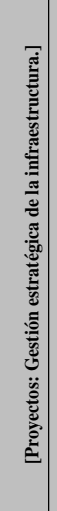 & 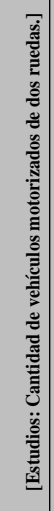 & 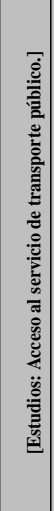 & 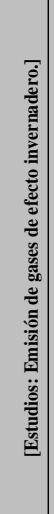 & 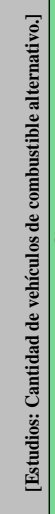 & \begin{tabular}{|c|c|} 
& \\
\\
Siempre \\
$\checkmark v$ \\
\\
\end{tabular} & $\begin{array}{c}\text { Casi } \\
\text { siempre } \\
\checkmark\end{array}$ & $\begin{array}{c}\text { Ocasional- } \\
\text { mente } \\
\checkmark \times\end{array}$ & $\begin{array}{c}\text { Casi } \\
\text { nunca } \\
\times\end{array}$ & $\begin{array}{c}\text { Nunca } \\
X \times\end{array}$ \\
\hline 1 & Verónica Delgado & ss. & $\checkmark v$. & $\checkmark$ & ss. & $\checkmark$ &,$\sqrt{x}$ &,$v x$ &,$v x$ &.$v x$ & $\checkmark$ &,$v \times$ &,$v x$ &.$v x$ &,$v x$ &,$v x$ &,$v x$ & 3 & 3 & 10 & 0 & 0 \\
\hline 2 & Diego Correa &,$v x$ &,$v x$ & $\checkmark$ & $\checkmark$ & $\checkmark$ & $\checkmark$ &,$v x$ & $\checkmark$ & $v x$ & $\checkmark$ & $\checkmark$ & $v x$ & $x$ & $\checkmark$ & $v x$ & $\checkmark$ & 0 & 9 & 6 & 1 & 0 \\
\hline 3 & Franklin Patiño &,$v x$ &,$v x$ &,$v x$ &.$v x$ &.$v x$ & $\mathrm{vx}$ &.$v x$ &.$v x$ &,$v \times$ &,$v x$ &,$v x$ &,$v x$ &,$v x$ &,$v x$ & $x$ & $x$ & 0 & 0 & 14 & 2 & 0 \\
\hline 4 & Guilherme Chalhoub & $x$ & $\checkmark$ & $\checkmark v$. & $\checkmark v$. & $\checkmark$ &,$v x$ & $\checkmark$ & $\checkmark$ &,$v \times$ & $a v$. & $\checkmark$ & su. &,$v x$ & $a v$. & $x$ &.$v x$ & 5 & 5 & 4 & 2 & 0 \\
\hline 5 & Juan Aviles & ss. & $v s$. &,$v x$ & $\checkmark$ &,$v x$ & $x$ & $x$ & $\checkmark$ & $\checkmark$ & $x$ & $x$ & $x$ & $v v$. &,$v x$ & $x$ & $x x$ & 3 & 3 & 3 & 6 & 1 \\
\hline 6 & Juan Diego Cueva & $\checkmark$ & , $x$ &,$v x$ &,$v x$ & $x$ & $x x$ & $\checkmark$ & $x$ & $x$ &,$v x$ &.$x x$ & $x$ & $x$ & $v x$ & $x x$ & $x$ & 0 & 2 & 5 & 6 & 3 \\
\hline 7 & Giorco & $\checkmark$ & vx & $x$ & $\checkmark$ &,$v x$ & $x$ & $x$ & $x$ & $x$ & $x x$ & $x$ & $x$ &,$v x$ & $x$ & $x x$ & $x x$ & 0 & 2 & 3 & 8 & 3 \\
\hline 8 & Jennyfer Flores &,$v x$ & $x$ & $\checkmark v$. & $x$ & $x$ & $x x$ & $v v$. & $x$ & $x$ & $x$ & $x x$ & $x$ & $x$ &,$v x$ & $x$ & $x x$ & 2 & 0 & 2 & 9 & 3 \\
\hline 9 & Xavier Contreras &,$v x$ &,$v \times$ &,$v x$ & $x$ & $x \times$ & $x \times$ &.$v x$ &,$v x$ & $x$ & $x x$ & $x x$ &,$v x$ &,$v x$ & $x x$ & $x$ & $x$ & 0 & 0 & 7 & 4 & 5 \\
\hline 10 & Andrés & , vx &.$v x$ & $x$ & $x$ & $x x$ & $x x$ & $\checkmark$ &.$v x$ & $x$ & $x x$ & $x x$ &,$v x$ &,$v x$ & $x x$ & $x$ & $x$ & 0 & 1 & 5 & 5 & 5 \\
\hline 11 & Hernán Quintuña &,$v x$ & $x$ & $x$ & $x$ & $x$ & $x$ & $x x$ &,$v x$ &,$v x$ & $x$ & $x x$ & $x x$ & $x$ & $x$ & $x x$ & $x x$ & 0 & 0 & 3 & 8 & 5 \\
\hline 12 & Francisco Darquea & sv. &,$v x$ & $v v$. & $x$ & $x$ & $x \times$ & 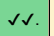 &,$v x$ & $x$ &,$v x$ & $x x$ & $x x$ & $x x$ & $x x$ & $x x$ & $x x$ & 3 & 0 & 3 & 3 & 7 \\
\hline 13 & Darwin Gordillo & $\checkmark$ & $x \times$ & $x x$ & $x$ & $x$ & $x x$ & $x$ & $x x$ &,$v \times$. & $x x$ & $x x$ & $x x$ & $\checkmark$ & $x x$ & $x x$ & $x x$ & 0 & 2 & 1 & 3 & 10 \\
\hline 14 & Caguana &,$v x$ & $. v \times$ &,$v x$ & $x x$ & $x x$ & $x \times$. & $x x$ & $x x$ & $x \times$. & $x x$ & $x x$ & $x x$ &.$v x$ & $x x$ & $x x$ & $x x$ & 0 & 0 & 4 & 0 & 12 \\
\hline 15 & Carlos Lemas & $\checkmark$ & $x x$ & $x$ & $x$ & $x x$ & $x \times$. & $x x$ & $x x$ & $x \times$. & $x x$ & $x x$ &.$x x$ & $x x$ & $x x$ & $x x$ & $x x$ & 0 & 1 & 0 & 2 & 13 \\
\hline
\end{tabular}

Nota: Proceso de selección de expertos en el área de movilidad en la ciudad de Cuenca. Fuente: Elaboración propia.

Por temas de pandemia, de los profesionales encuestados en forma digital, se tuvo un contacto directo e indirecto con quienes estuvieron muy gustosos en colaborar y además 
cuentan con gran conocimiento sobre la movilidad. Se presentó ante ellos los indicadores propuestos en la presente investigación, estos fueron puntuados en base a la suficiencia, claridad, relevancia y coherencia que presentaban, obteniendo los resultados que se observa en la figura 7, con un alto, moderado o bajo nivel de cumplimiento.

\section{Figura 7}

Calificación de indicadores en base de a criterios de suficiencia, claridad, relevancia y coherencia para una movilidad sostenible en la ciudad de Cuenca

Calificación de indicadores propuestos para una movilidad sostenible, en base a criterios de: suficiencia, claridad, coherencia y relevancia.

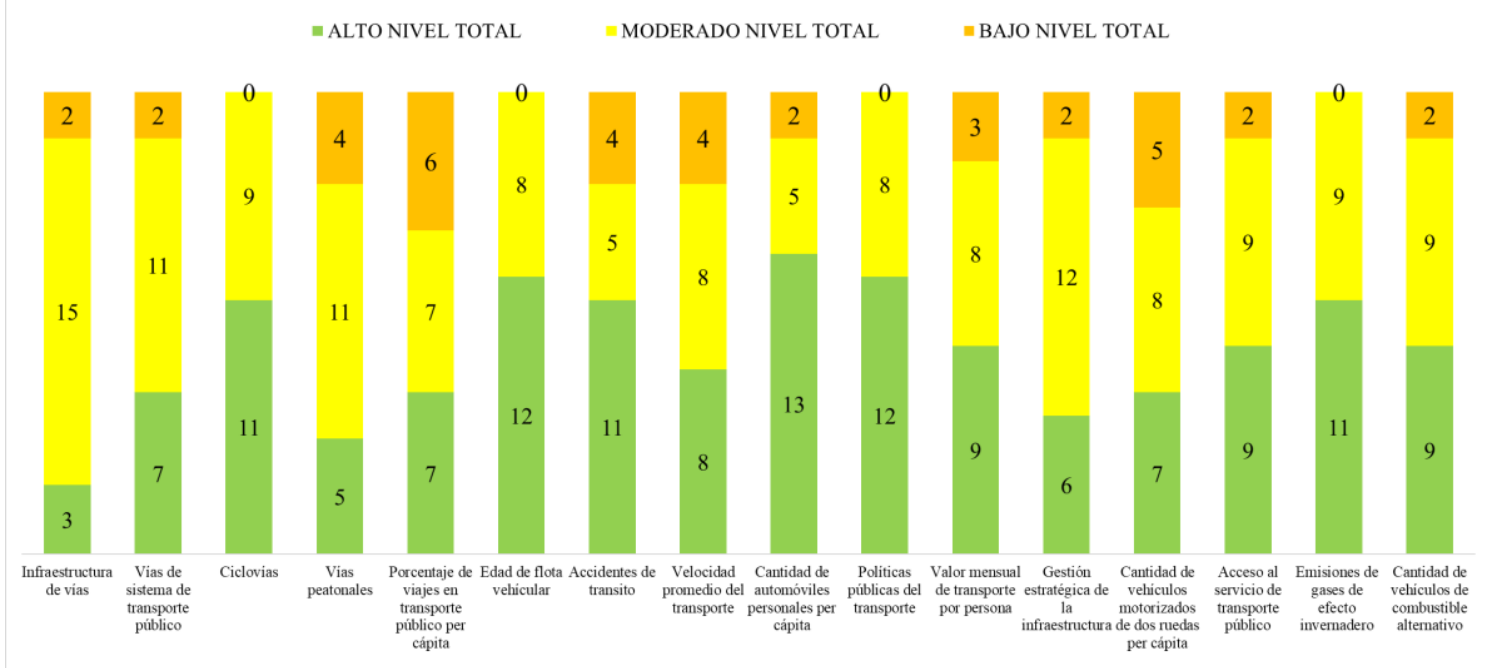

Nota: Puntaje obtenido por cada indicador en base a la calificación de los expertos.

Se puede observar en la figura 7, en base a la puntuación de cada indicador que la movilidad juega un papel importante en la ciudad de Cuenca, según la apreciación de los expertos, el indicador que analiza la infraestructura de vía no cuenta con una gran calificación de alto nivel de acuerdo a suficiencia, claridad, relevancia y coherencia, caso contrario sucede en cambio con el indicador de la cantidad de automóviles personales per cápita, que cuenta con un alto número de calificaciones en un alto nivel.

Finalmente, se realizó una evaluación de 3 indicadores, el de mayor calificación con un alto nivel, y dos adicionales considerados importantes en la propuesta, obtenido los resultados que se observan en la tabla 2. 


\section{Conciencia}

Tabla 2

Evaluación de indicadores de movilidad sostenible para la ciudad de Cuenca - Ecuador

\begin{tabular}{|c|c|c|c|c|c|c|c|c|}
\hline \multirow[b]{2}{*}{ No. } & \multirow[b]{2}{*}{ Indicador } & \multirow[b]{2}{*}{ Unidad } & \multicolumn{3}{|c|}{ Niveles de Exigencias } & \multirow{2}{*}{$\begin{array}{l}\text { Indicador } \\
\text { medido }\end{array}$} & \multirow[b]{2}{*}{$\begin{array}{l}\text { Valor } \\
\text { actual }\end{array}$} & \multirow[b]{2}{*}{ Referencia } \\
\hline & & & Sostenible & $\begin{array}{c}\text { Medianamente } \\
\text { sostenible }\end{array}$ & $\begin{array}{c}\text { No } \\
\text { sostenible }\end{array}$ & & & \\
\hline 3 & Ciclovías & $\mathrm{Km}$ & $>25$ & $15-25$ & $<15$ & 0,001 & 52,73 & $\begin{array}{l}\text { http://www.cuenca.gob. } \\
\text { ec/?q=content/planes- } \\
\text { pdot-pugs-2020 }\end{array}$ \\
\hline 7 & $\begin{array}{l}\text { Accidentes } \\
\text { de tránsito. }\end{array}$ & Cantidad & $<0,1$ & $0,1-0,2$ & $>0,2$ & 0,163 & 163 & $\begin{array}{l}\text { https://www.emov.gob.e } \\
\text { c/gestion-efectiva-de-la- } \\
\text { emov-ep-genera- } \\
\text { reduccion-de-indice-de- } \\
\text { accidentabilidad-en- } \\
\text { cuenca/ }\end{array}$ \\
\hline 9 & $\begin{array}{l}\text { Cantidad de } \\
\text { automóviles } \\
\text { personales } \\
\text { per cápita }\end{array}$ & Cantidad & $<0,3$ & $0,3-0,4$ & $>0,4$ & 0,170 & 98527 & $\begin{array}{l}\text { https://www.emov.gob.e } \\
\text { c/mas-de-75-000- } \\
\text { vehiculos-fueron- } \\
\text { matriculados-en-cuenca- } \\
\text { durante-el- } \\
\text { 2021/\#: : } \\
\text { A1s\%xt=M\%C3\% } \\
\text { 20veh\%C3\% } \% 2075.000 \% \\
\text { 20fueron\%20matriculad } \\
\text { os\%20en\%20Cuenca\%2 } \\
\text { 0durante\%20el\%202021 } \\
\text {,-Oct\%2005\%202021 }\end{array}$ \\
\hline
\end{tabular}

De la evaluación de los indicadores en la tabla 4, en base a la referencia de datos publicados en la página del organismo regulador para la ciudad de Cuenca, EMOV (Empresa de Pública de Movilidad, Tránsito y Transporte de Cuenca), el indicador de ciclovías no es satisfactorio, ya que se obtuvo un valor de 0.001. Esto se debe a que en la ciudad existe solamente $52.73 \mathrm{Km}$ de infraestructura vial dedicadas a la bicicleta como se observar en la figura 8 . 


\section{Figura 8}

\section{Mapa infraestructura para bicicletas Cuenca - Ecuador}
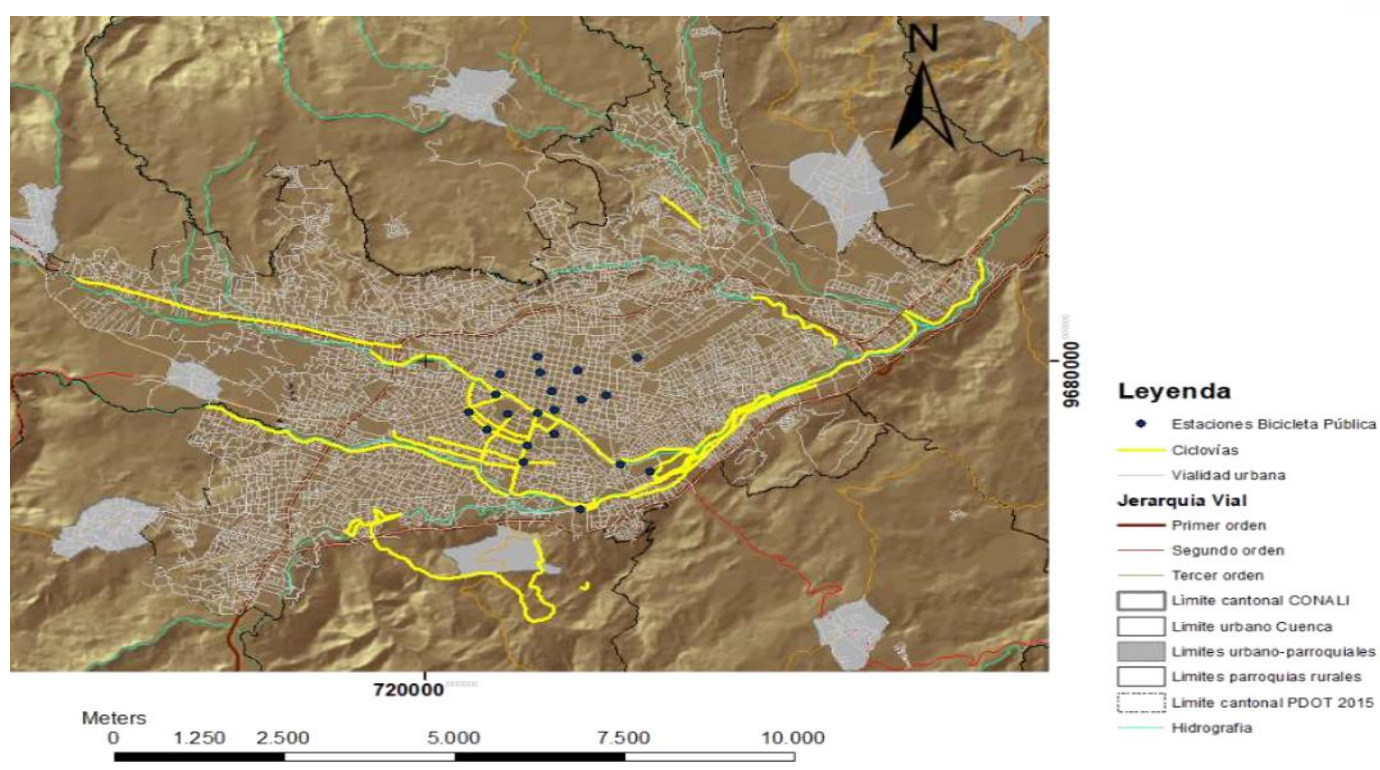

Nota: Mapa de infraestructura para bicicletas en la ciudad de Cuenca.

Fuente: PDOT-GAD Municipal del Cantón Cuenca (EMOV EP, 2019)

Para el indicador de accidentes de tránsito, Cuenca tuvo una reducción para el año 2021, según la referencia, se muestra el valor del indicador con 0.163 consiguiendo el mismo ser medianamente sostenible. Y finalmente para el indicador referente a la cantidad de automóviles personales per cápita con mayor calificación de alto nivel por los expertos, al obtener un valor de 0.170 cumple con el criterio de sostenibilidad.

\section{Discusión}

Entidades encargadas de la movilidad, para el caso de la ciudad de Cuenca, la EMOV (Empresa Pública de Movilidad, Tránsito y Transporte de Cuenca) son las que deberían adoptar estas medidas entre sus políticas, teniendo así una base de indicadores medibles periódicamente, para posterior aplicar a certificaciones de proyectos de movilidad sostenible obtenido los beneficios que estos conllevan.

\section{Conclusiones}

- Muchos de los indicadores obtenidos tienen una estrecha relación entre ellos, pero cada uno cuenta con una evaluación cuantitativa diferente, para lo cual se deberá establecer límites según la ciudad en análisis. 
- En base a la evaluación de los 3 indicadores de movilidad propuesto para la ciudad de Cuenca, se tuvo que en el caso del indicador de ciclovías no existe sostenibilidad, para el indicador de accidentes de tránsito es medianamente sostenible y finalmente para el indicador de cantidad de automóviles personales, la ciudad cuenta con una movilidad sostenible de acuerdo con los límites planteados en la bibliografía.

- Basados en las observaciones emitidas por los expertos en la calificación de los indicadores, ellos toman en cuenta el tema de que en la ciudad de Cuenca, se tiene vías de cuarto orden o incluso algunas aún no se encuentran catastradas, por lo que la evaluación del indicador relacionado a este tema no sería la correcta, de igual manera sucede con los vehículos, existen muchos que no se encuentran con permiso de circulación o que la matriculación lo hacen en otro lugar, entonces la evaluación de acuerdo al criterio establecidos en la guías o herramientas de certificación no va a ser el real.

- Otros expertos tienen cierta discrepancia con respecto al método de evaluación y a los rangos de medición de los indicadores, generando así diferentes criterios. Por lo considera el autor como el punto de partida a futuras investigaciones las que definirán rangos de calificación y de acuerdo con las características de la ciudad, definir metodologías claras y establecer criterios de evaluación.

\section{Agradecimientos}

El presente artículo es parte del trabajo de investigación y titulación del Programa de Maestría en Construcción con Mención en Administración de la Construcción Sustentable de la Universidad Católica de Cuenca, vinculados al Proyecto de Investigación: INDICADORES DE SOSTENIBILIDAD URBANA PARA LA CIUDAD DE CUENCA - ECUADOR, por ello agradecemos a todos y cada uno de los instructores pertenecientes a los grupos de investigación; Ciudad, Ambiente y Tecnología (CAT), y Sistemas embebidos y visión artificial en ciencias, Arquitectónicas, Agropecuarias, Ambientales y Automática (SEVA4CA), por los conocimientos e información brindados para la elaboración del trabajo.

\section{Referencias bibliográficas}

Plan de desarrollo y ordenamiento territorial del cantón Cuenca. [PDOT]. (2021, 17 de marzo). Memoria técnica de actualización de desarrollo y ordenamiento territorial. https://planificacion.cuenca.gob.ec/bc-articulo/plan-de-desarrollo-y-ordenamientoterritorial-de-cuenca-0

Ahvenniemi, H., Huovila, A., Pinto-Seppä, I., \& Airaksinen, M. (2017). What are the differences between sustainable and smart cities? Cities, 60, 234-245. 
BREEAM. (2012). BREEAM Communities. Technical Manual SD202 - 0.1:2012. https://www.breeam.com/communitiesmanual/content/resources/otherformats/outp ut/10_pdf/20_a4_pdf_screen/sd202_breeam_communities_1.2_screen.pdf

Brundtland, G. H. (1987). Informe de la Comisión Mundial sobre Medio Ambiente y el Desarrollo: Nuestro futuro común. http://www.ecominga.uqam.ca/PDF/BIBLIOGRAPHIE/GUIDE_LECTURE_1/C MMAD-Informe-Comision-Brundtland-sobre-Medio-Ambiente-Desarrollo.pdf

CASBEE for cities (2012). Comprehensive Assessment System for Built Environment Efficiency. https://www.ibec.or.jp/CASBEE/english/download.htm

Cervero, R. (2013). Linking urban transport and land use in developing countries. Journal of Transport and Land Use, 6(1), 7-24.

Damidavičius, J., Burinskiene, M., \& Antuchevičiene, J. (2020). Assessing sustainable mobility measures applying multicriteria decision making methods. Sustainability (Switzerland), 12(15), 1-14.

Engström, G., Gars, J., Jaakkola, N., Lindahl, T., Spiro, D., \& Van Benthem, A. A. (2002). Informe de la Cumbre Mundial sobre el Desarrollo Sostenible. In Environmental and Resource Economics.

Escobar-Pérez, J., \& Cuervo-Martínez, Á. (2008). Validez de contenido y Juicio de expertos: Una Aproximación a su utilización. Avances En Medición, 6, $27-36$.

Flores Juca, E., García Navarro, J., Chica Carmona, J., \& Mora Arias, E. (2017). Identification and analysis of sustainability indicators for mobility. Estoa, 6(11), 99109.

Gillis, D., Semanjski, I., \& Lauwers, D. (2015). How to Monitor Sustainable Mobility in Cities? Literature Review in the Frame of Creating a Set of Sustainable Mobility Indicators. Sustainability, 8(29), 1-30.

Gudmundsson, H., Hall, R. P., Marsden, G., \& Zietsman, J. (2016). Springer Texts in Business and Economics Sustainable Transportation. Editorial Springer.

Guzman, L. A., Arellana, J., \& Alvarez, V. (2020). Confronting congestion in urban areas: Developing Sustainable Mobility Plans for public and private organizations in Bogotá. Transportation Research Part A: Policy and Practice, 134(March 2019), $321-335$.

Ilustre Municipalidad de Cuenca. (2015). Plan de Movilidad y Espacios Públicos de Cuenca,

2015-2025. 
https://hackathon.cedia.edu.ec/dmdocuments/INNOVACION/RETO/www.cuenca. gob.ec-1.pdf

ISO 37120 standard on city indicators-how they help city leaders set tangible targets, including service quality and quality of life. (2014). Centre for Livable Cites, Singapore. https://www.iso.org/standard/62436.html

Jain, D., \& Tiwari, G. (2017). Sustainable mobility indicators for Indian cities_Selection methodology and application. Ecological Indicators, 79(10), 310-322.

LEED v4. 1 ciudades y Comunidades (2020, 10 de abril). Planificar y Diseñar. https://es.scribd.com/document/524123410/LEED-v41-CC-Plan-and-DesignGuide-Spanish-Jun-2020

Manzanares Garmendia, G. (2020). Desarrollo sostenible y políticas públicas: enfoque de la ONU y ecología política. Revista Ciencia Jurídica y Política, 6(12), 73-87.

Mascarenhas, A., Nunes, L. M., \& Ramos, T. B. (2015). Selection of sustainability indicators for planning: Combining stakeholders' participation and data reduction techniques. Journal of Cleaner Production, 92, 295-307.

Guía metodológica BID. (2016). Guía Metodológica iniciativa ciudades emergentes y sostenibles.

https://publications.iadb.org/publications/spanish/document/Gu\%C3\%ADa-

Metodo1\%C3\%B3gica-Programa-de-Ciudades-Emergentes-y-Sostenibles-Terceraedici\%C3\%B3n.pdf

Mozos-Blanco, M. Á., Pozo-Menéndez, E., Arce-Ruiz, R., \& Baucells-Aletà, N. (2018). The way to sustainable mobility. A comparative analysis of sustainable mobility plans in Spain. Transport Policy, 72(July), 45-54.

La Agenda 2030 y sus Objetivos de Desarrollo Sostenible. (2018). Una oportunidad para América Latina $\quad$ El Caribe. https://repositorio.cepal.org/bitstream/handle/11362/40155/24/S1801141_es.pdf

UN-Habitat. Sustainable Development Goal 11 - Make cities and human settlements. Monitoring framework. (2019). A Guide to Assist National and Local Governments to Monitor and Report on SGD Goal 11+ Indicators. https://sustainabledevelopment.un.org/sdg11

United Nations ESCAP. (2020). Assessment of Urban Transport and Impacts of COVID19 on Mobility Data Collection Guideline. https://repository.unescap.org/handle/20.500.12870/120?locale-attribute=es 


\section{Conciencia}

\section{Conflicto de intereses}

Los autores deben declarar si existe o no conflicto de intereses en relación con el artículo presentado.

El artículo que se publica es de exclusiva responsabilidad de los autores y no necesariamente reflejan el pensamiento de la Revista Conciencia Digital.

\section{Ciencia \\ LDigital}

El artículo queda en propiedad de la revista y, por tanto, su publicación parcial y/o total en otro medio tiene que ser autorizado por el director de la Revista Conciencia Digital.
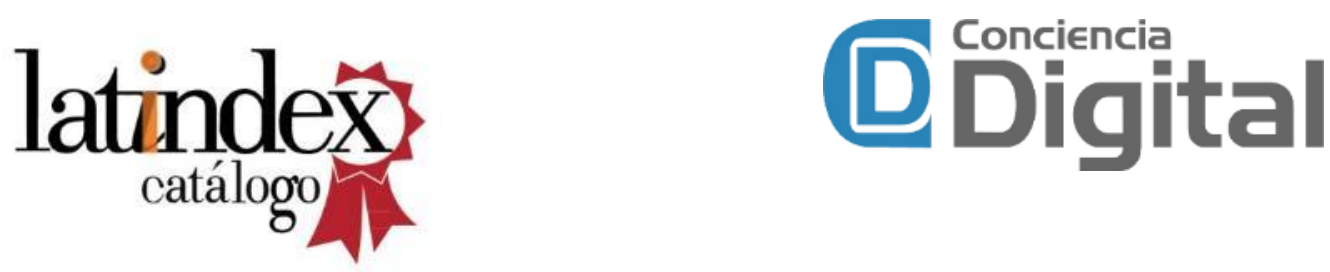

Indexaciones

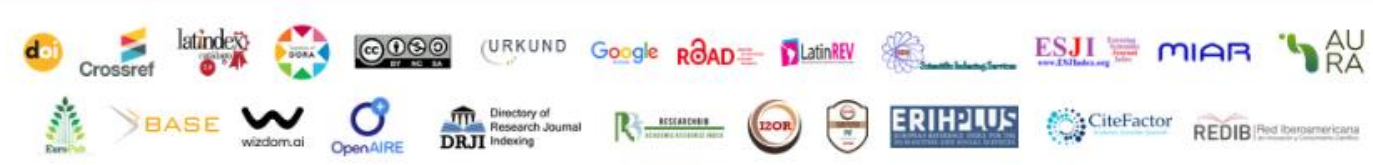

